\title{
Exploring patterns of recurrent melanoma in Northeast Scotland to inform the introduction a digital self-examination intervention
}

\author{
Rhona Auckland ${ }^{1}$, Patrick Wassell ${ }^{2}$, Susan Hall ${ }^{3 *}$, Marianne C Nicolson ${ }^{4}$ and Peter Murchie ${ }^{3}$
}

\begin{abstract}
Background: Melanoma incidence is growing and more people require follow-up to detect recurrent melanoma quickly. Those detecting their own recurrent melanoma appear to have the best prognosis, so total skin self examination (TSSE) is advocated, but practice is suboptimal. A digital intervention to support TSSE has potential but it is not clear which patient groups could benefit most. The aim of this study was to explore cutaneous melanoma recurrence patterns between 1991 and 2012 in Northeast Scotland. The objectives were to: determine how recurrent melanomas were detected during the period; explore factors potentially predictive of mode of recurrence detection; identify groups least likely to detect their own recurrent melanoma and with most potential to benefit from digital TSSE support.

Methods: Pathology records were used to identify those with a potential recurrent melanoma of any type (local, regional and distant). Following screening of potential cases available secondary care-held records were subsequently scrutinised. Data was collected on demographics and clinical characteristics of the initial and recurrent melanoma. Data were handled in Microsoft Excel and transported into SPSS 20.0 for statistical analysis. Factors predicting detection at interval or scheduled follow-up were explored using univariate techniques, with potentially influential factors combined in a multivariate binary logistic model to adjust for confounding.

Results: 149 potential recurrences were identified from the pathology database held at Aberdeen Royal Infirmary. Reliable data could be obtained on 94 cases of recurrent melanoma of all types. 30 recurrences (31.9\%) were found by doctors at follow-up, and $64(68.1 \%)$ in the interval between visits, usually by the patient themselves. Melanoma recurrences of all types occurring within one-year were significantly more likely to be found at follow-up visits, and this remained so following adjustment for other factors that could be used to target digital TSSE support.
\end{abstract}

Conclusions: A digital intervention should be offered to all newly diagnosed patients. This group could benefit most from optimal TSSE practice.

Keywords: Melanoma recurrence, Self-detected, Follow-up, Skin self-examination, Education

\section{Background}

Melanoma incidence has risen over the last 50 years, and disproportionately affects younger people [1]. Around three times as many cases were reported in 2000 than in 1970 and it is now the sixth commonest cancer in the UK $[2,3]$. Scottish guidelines recommend that people treated for $\mathrm{cu}-$ taneous melanoma receive stuctured follow-up consisting

\footnotetext{
* Correspondence: s.hall@abdn.ac.uk

${ }^{3}$ Centre of Academic Primary Care - Division of Applied Health Sciences, University of Aberdeen, Polwarth Building, Foresterhill, Aberdeen AB25 2ZD, UK Full list of author information is available at the end of the article
}

of regular physical examination by a specialist without blood tests or imaging unless subsequently indicated [4]. Follow-up aims to detect melanoma recurrences early and expedite secondary care access if necessary and its delivery is becoming increasingly burdensome to healthcare systems [5-7]. Many recurrences are detected in the interval between structured follow-up visits leading many to question it value $[8,9]$. On the other hand there is evidence that most early recurrences (within two years) are not self-detected but found at scheduled follow-up appointments [8,10-13]. This is important since there is evidence of superior 


\section{Table 1 Melanoma recurrence data sheet}

Surname:

Forename(s):

$\mathrm{CHI}:$

Occupation:

Date of primary melanoma diagnosis:

Melanoma type:

Stage of melanoma:

Tumour thickness (mm):

Prognostic features:

Tumour anatomical location:

Method/Details of treatment:

Details on how the melanoma was picked up:

Date of excision biopsies:

Details of prescribed follow-up program:

Date of recurrence diagnosis:

Nature of recurrence: local, regional, distant)

Melanoma type:

Melanoma stage:

Tumour thickness:

Prognostic features:

Tumour anatomical location:

How was the recurrence detected:

If other, please state:

If self- detected, which of the following apply:

Recurrence found via;

Regression;

Primary biopsy;
Self - detected

GP

Dermatologist

Follow-up appointment

Other

DOB:

Gender:

Ethnicity:

Breslow depth;

Clark level;

Ulceration;

Lymph node involvement;

Tumour vascularity;

lymphovascular invasion;

Mitotic rate;

Microsatellites;

Tumour-infiltrating lymphocytes;

Lactate dehydrogenase serum level;

Secondary biopsy;

Punch/Shave biopsy;

Breslow depth;

Clark level;

Please Tick

Please Tick:

Routine self-examination

Accidental find

Aid of partner/Acquaintance 
survival rates of those who self-detected their recurrence appear to have superior survival, corresponding with with findings that regular total skin self examinations (TSSE) in people treated for primary cutaneous melanoma can reduce mortality rates by as much as $63 \%[13,14]$.

Despite this TSSE education and practice appear suboptimal with $70 \%$ of American melanoma patients indicating that they had never been advised to do it [15]. There is good reason to suggest that similar statistics would be found in North East Scotland (NES). Where interventions to improve TSSE have been tried, results have been disappointing and those who were educated by brochure or video demonstrations only reported increased TSSE practice for 3-7 months, with overall participation returning to the baseline by 12 months [16-18]. Despite this it is encouraging that educational interventions could achieve $17-50 \%$ increases in TSSE practice, albeit in the short-term. Further, stabilisation of mortality in younger patients (aged 25-44), despite increasing incidence, is thought to result from increased public awareness and TSSE promotion $[1,16,19,20]$. Similarly higher survival and higher TSSE rates are observed in less deprived people [15]. All this supports the view that TSSE is worth doing. Some suggest that digital interventions could promote and sustain TSSE practice [18-20]. Such interventions however, are likely to be expensive to develop and implement so should be targeted at those with the greatest potential to benefit, information which the current studies do not provide.

We are developing a digital intervention to promote and prompt TSSE in Northeast Scotland. We wished to explore patterns of all types of melanoma recurrence within the region over recent years to determine when, and to which patients, this intervention should be targeted.

\section{Methods}

\section{Study approval}

Formal approval for this study was granted on $10^{\text {th }}$ October 2012 by the Quality, Governance and Risk Unit (Clinical Effectiveness Team) of NHS Grampian (project ID 2483).

\section{Identifying recurrences}

A data-base maintained by the Department of Pathology, NHS Grampian was scrutinised to identify melanoma patients from Northeast Scotland potentially diagnosed with any type of recurrence between August 1992 and September 2012.

\section{Data collection}

A data collection sheet was constructed (Table 1) and used to abstract data from the secondary care-held medical records of eligible and available cases at the medical records department at Aberdeen Royal Infirmary.

The following data were abstracted (Table 2):

i) Demographics: gender; date of birth; age at diagnosis; age at recurrence; postcode. Postcode was subsequently use to define deprivation and rurality [21,22].

ii) Clinical details of the initial melanoma including: melanoma type; Breslow thickness; anatomical location.

iii) Type of recurrence: local; regional; distant.

iv) Recurrence pathway: time to recurrence; mode of detection (self detected and reported during interval; found at follow-up clinic by clinician; emergency admission during interval; other).

Data were entered handled using a Microsoft Excel worksheet. After scrutiny for errors they were read into SPSS version 20 for further statistical analysis.

\section{Statistical analysis}

\section{Demographic and clinical characteristics}

Basic descriptive statistics of the sample were prepared. Demographics characteristics, clinical characteristics of the primary melanoma and details of the recurrence and its detection pathway were explored.

\section{Categorising variables}

To conduct meaningful univariate analysis on this relatively small sample was challenging. A number of categorical variables were created from the data to address this. After discussion amongst the authors a binary outcome variable was created distinguishing those patients who had their recurrent melanoma detected de-novo at the follow-up clinic versus those in whom the presentation of recurrence had occurred in the interval between scheduled follow-up visits. Arguably, the former group are those who stand most to gain from interventions to promote total skin self examination. 
Table 2 Clinicopathological Characteristics; descriptive statistics in relation to frequency within sample of 94 patients

Frequency:

Demographics:

Frequency:

Gender;

Female

$37(39.4 \%)$

Male

Deprivation score;

1 (most deprived)

2

3

4

5(least deprived)

Six-fold rurality score;

Urban

Rural

Type of recurrence

Local

$21(22.3 \%)$

Regional

Distant

Details of first primary:

Age at diagnosis;

$<=50$ years

$19(20.2 \%)$

51-70 years

$71+$ years

Unknown

Melanoma type of first primary;

Superficial spreading

Nodular malignant

Lentigo maligna

Acral lentiginous

Other

Location of first primary;

Lower limbs

Trunk

Upper limbs

Head and neck

Mucus membranes

Eye

Breslow thickness of first primary;

$<0.75 \mathrm{~mm}$

$0.76-4.00 \mathrm{~mm}$

$>4.00 \mathrm{~mm}$

Unknown

$5(5.3 \%)$

9 (9.6\%)

$22(23.4 \%)$

$27(28.7 \%)$

31 (33.0\%)

$43(45.6 \%)$

$51(54.4 \%)$

$48(51.1 \%)$

$25(26.6 \%)$
Table 2 Clinicopathological Characteristics; descriptive statistics in relation to frequency within sample of 94 patients (Continued)

Details of recurrence:

\section{Age at recurrence;}

$<=50$ years

$14(14.9 \%)$

51-70 years

40 (42.6\%)

$71+$ years

$40(42.6 \%)$

Time to recurrence;

0-12 months

$27(28.7 \%)$

13-24 months

$28(29.8 \%)$

25-36

$11(11.7 \%)$

$37-48$

$5(5.3 \%)$

49-60

$7(7.4 \%)$

$5+$ years

$15(16.0 \%)$

Unknown

$1(1.1 \%)$

How recurrence was detected;

Self-detected and reported during interval $\quad 45$ (48.9\%)

New finding at follow-up clinical $\quad 30$ (31.9\%)

Emergency admission with symptoms in interval $\quad 11(11.7 \%)$

Other

$5(5.3 \%)$

Seven predictor variables were then created. These were selected on the basis that there was evidence for, or it seemed plausible that, they could be influential on whether recurrent melanoma was detected at scheduled follow-up or during the interval between scheduled follow-up appointments. Candidates were classified by gender, 5-fold deprivation score and 2-fold rurality score. With reference to recurrent melanoma, variables including age ( $<65$ versus $\geq 65$ years) time to recurrence $(<1$ year versus $\geq 1$ year) were considered and details of the initial melanoma (subtype, Breslow thickness $(<4 \mathrm{~mm} ; \geq 4 \mathrm{~mm})$ ) [8].

\section{Univariate analysis}

The outcome variable (detected at scheduled follow-up or not) was cross-tabulated with each of the six predictor variables. The chi-squared test was used to ascertain if the six factors were significantly associated with follow-up versus interval detection, with statistical significance being designated as a $\mathrm{p} \leq 0.05$. The strength of the relationship between the outcome and each of the six predictor variables was also explored univariately using binary logistic regression to generate odds ratios and 95\% CIs.

\section{Multivariate analysis}

A multivariate binary logistic regression model was then constructed including all factors found to be significantly predictive of follow-up versus interval recurrence detection, as well as those factors that could conceivably inform targeting of a digital intervention to support 
TSSE (Breslow thickness; time to recurrence; age at recurrence; rurality; deprivation score).

\section{Results}

Included and excluded cases

149 potential cases of any type of recurrent melanoma were identified (Figure 1). Eighteen people had died and notes were not available and four sets of notes could not be located. Thirty-one people had recurrent melanoma with no record of a prior primary. Two further apparent recurrences proved to be new basal cell carcinomas on review of pathology data. This resulted in 55 exclusions and a final sample of 94 cases for analysis.

\section{Demographic and clinical characteristics}

Demographics and characteristics of primary melanomas are shown in Table 2. The sample included 21 (22.3\%) local, 48 (51.1\%) regional and 25 (26.6\%) distant recurrences. Mean age at the time of recurrence was 65 years. Forty-five recurrences $(58.5 \%)$ had presented within two years of diagnosis. Thirty (31.9\%) cases were detected at scheduled melanoma follow-up appointments. The remaining 64 (69.1\%) were detected as interval events with 45 (48.9\%) being obviously self detected, 11 (11.7\%) being emergency admissions to hospital with metastatic disease and five $(5.3 \%)$ being detected through another route, mostly incidental findings.

\section{Univariate analysis}

Those variables deemed most likely to affect whether a recurrence was found at follow-up or self detected in the interval were explored univariately with the chi-squared test (Table 3). The most striking finding was earlier recurrences were significantly more to be detected at structured follow-up. Of recurrences within a year of diagnosis 51.9\% were detected at follow-up, while only $23.9 \%$ of later recurrences were detected at follow-up. No other potential predictors of recurrence detection location were significant univariately.

\section{Logistic regression}

A binary logistic regression of mode of recurrence detection (at follow-up versus interval) was conducted (Table 4). Those who had a recurrence within one year were 3.433 (95\% CIs 1.340-8.796) times more likely to be detected at structured follow up than in the interval. Following adjustment for other potential explanatory variables time to recurrence remained the only potentially explanatory variable significantly associated with mode of recurrence detection (OR2.891 (95\% CIs 1.082-7.720).

\section{Discussion}

\section{Summary of key findings}

Approximately one-third of recurrent melanomas in this small sample were detected at a routine scheduled followup appointment. Of those presenting in the interval, the majority were detected by the patient themselves. Of potential predictors of mode of melanoma recurrence only time to recurrence was statistically significant, with people being much less likely to detect their own recurrence within the first year since diagnosis.

\section{Context with other literature}

In this sample nearly one third of melanoma recurrences were detected at scheduled follow-up appointments. This accords with previous findings that $26-45 \%$ of melanoma recurrences are found at scheduled follow-up by a clinician $[8,9,13]$. Of the remaining two thirds the majority

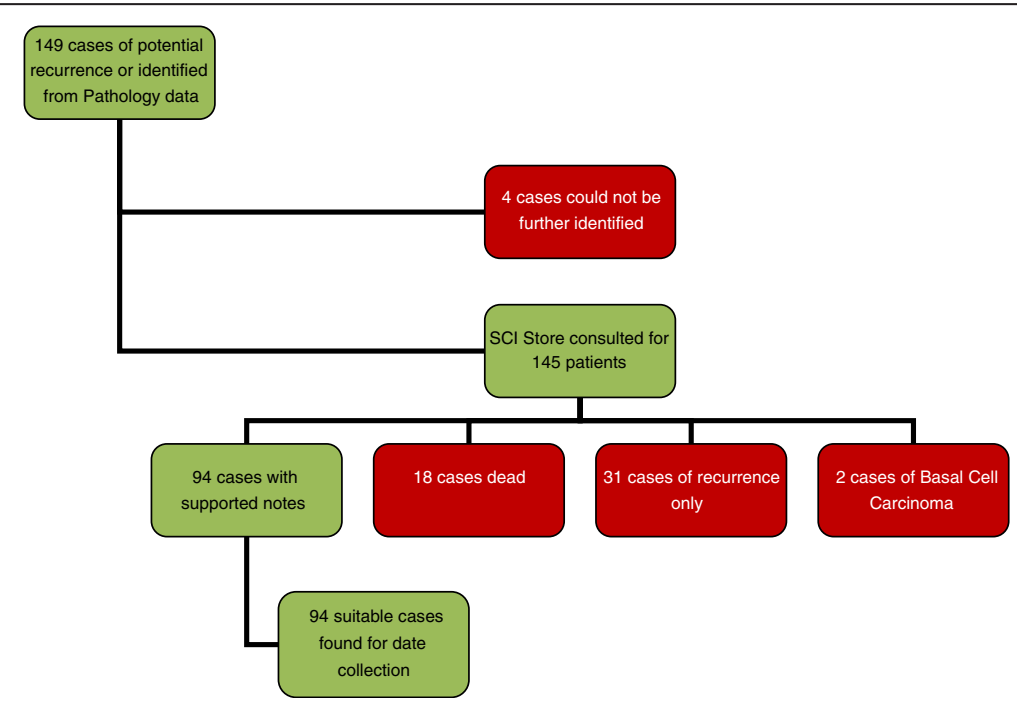

Figure 1 Study flow. 
Table 3 Cross tab data analysis; determining the significance of categorised variables on likelihood to present at follow-up or in interval

\begin{tabular}{|c|c|c|c|}
\hline Descriptive statistic: & $\begin{array}{l}\text { Detected at } \\
\text { follow-up: }\end{array}$ & $\begin{array}{l}\text { Detected } \\
\text { in interval: }\end{array}$ & p. value: \\
\hline Gender; & & & .413 \\
\hline Female & $8(21.6 \%)$ & $29(78.4 \%)$ & \\
\hline Male & $15(26.3 \%)^{\prime}$ & $42(73.7 \%)$ & \\
\hline Deprivation score; & & & .341 \\
\hline 1 (most deprived) & $3(60.0 \%)$ & $2(40.0 \%)$ & \\
\hline 2 & $3(33.3 \%)$ & $6(66.7 \%)$ & \\
\hline 3 & $6(27.3 \%)$ & $16(72.7 \%)$ & \\
\hline 4 & 7 (25.9\%) & $20(74.15)$ & \\
\hline 5(least deprived) & $4(12.9 \%)$ & $27(87.1 \%)$ & \\
\hline Rurality; & & & .571 \\
\hline Urban & $14(32.6 \%)$ & 29 (67.4\%) & \\
\hline Rural & $9(17.6 \%)$ & $42(82.4 \%)$ & \\
\hline Melanoma type; & & & .964 \\
\hline Superficial spreading & 7 (25.9\%) & $20(74.1 \%)$ & \\
\hline Nodular malignant & $8(26.7 \%)$ & $22(73.3 \%)$ & \\
\hline Lentigo maligna & $1(20.0 \%)$ & $4(80.0 \%)$ & \\
\hline Acral lentiginous & $1(20.0 \%$ & $4(80.0 \%)$ & \\
\hline Other & $6(22.2 \%)$ & $21(77.8 \%)$ & \\
\hline Breslow thickness; & & & .252 \\
\hline $4 \mathrm{~mm}$ or less & $11(20.0 \%)$ & 44 (80.0\%) & \\
\hline$>4 \mathrm{~mm}$ & $12(30.8 \%)$ & $27(69.2 \%)$ & \\
\hline Age at Dx; & & & .142 \\
\hline Up to 65 years & $12(25.0 \%)$ & $36(75 \%)$ & \\
\hline 65 years and above & $18(39.1 \%)$ & $28(60.9 \%)$ & \\
\hline Time to recurrence; & & & .008 \\
\hline 12 months or less & $14(51.9 \%)$ & $13(48.1 \%)$ & \\
\hline Beyond 12 months & 16 (23.9\%) & $51(76.2 \%)$ & \\
\hline
\end{tabular}

were detected by the patients themselves in the interval between follow-up visits. Again, this accords with previous findings which report that between $47-68 \%$ of recurrent melanomas are self-detected [8,9]. That almost two thirds of people in this sample, and similar proportions in previous studies, have detected their own recurrence supports the view expressed by some that the resources devoted to melanoma follow-up should be used to educate patients in the practice of TSSE, rather than being spent on blood tests and clinical imaging [8-10,12,23,24]. The 16 patients with emergency or incidental presentation of their melanoma might all represent missed opportunities to self-detect recurrent melanoma with effective TSSE. A digital method to prompting and support TSSE appears timely and warranted $[13,14]$. Implementing novel technological approaches to cancer can be costly [25]. Our data suggest
Table 4 Binary logistic regression; odds ratio of having recurrence detected at routine follow-up versus interval, based on key variables and individual factors

\begin{tabular}{|c|c|c|c|}
\hline & \multirow{2}{*}{$\begin{array}{l}\text { Odds } \\
\text { ratio: }\end{array}$} & \multicolumn{2}{|c|}{$95 \% \mathrm{Cl}$ for odds ratio } \\
\hline & & Lower & Upper \\
\hline Time to recurrence $(<1$ year) & 3.433 & 1.340 & 8.796 \\
\hline Gender (male) & 1.295 & .486 & 3.449 \\
\hline Age (>65 years) & 1.351 & .518 & 3.523 \\
\hline \multicolumn{4}{|l|}{ Melanoma type } \\
\hline (Nodular vs. superficial) & .842 & .274 & 2.586 \\
\hline (Lentigo vs. superficial) & .632 & .088 & 4.532 \\
\hline (Acral vs. superficial) & 1.684 & .162 & 17.516 \\
\hline (Other vs. superficial) & .842 & .267 & 2.660 \\
\hline Breslow thickness (Thicker (>4 mm)) & 1.667 & .694 & 4.004 \\
\hline Rurality (Urban) & 1.286 & .539 & 3.067 \\
\hline Deprivation (Deprived) & 1.750 & .548 & 5.591 \\
\hline \multicolumn{4}{|l|}{ Adjusted binary logistic regression: } \\
\hline Time to recurrence & 2.891 & 1.082 & 7.720 \\
\hline Age & 1.714 & .666 & 4.415 \\
\hline Breslow thickness & 1.443 & .573 & 3.635 \\
\hline Rurality & 1.377 & .527 & 3.596 \\
\hline Deprivation & 1.626 & .454 & 5.816 \\
\hline
\end{tabular}

Adjusted Binary Logistic Regression; adjusted for variables we deemed could influence our values.

this cost could be mitigated by targeting those most likely to benefit.

Previous researchers have established that adjusting to a diagnosis of cancer takes time [26]. It is striking that those within a year of diagnosis were less likely to detect their own recurrent melanoma. This finding fits well with the notion of taking time to adjust to melanoma and the practice of TSSE [27]. Perhaps those in the immediate post-diagnostic period have most to gain from intensive support with respect to TSSE. In this context it is reassuring to note that previous interventions to promote TSSE, demonstrate it can be increased by well designed educational interventions [17-20].

\section{Strengths and limitations}

Our study contributes knowledge to an under-researched area, the epidemiology of recurrent melanoma. Our study was rigorous and based on high quality clinical databases and medical records. This was not an attempt to fully describe the epidemiology of recurrent melanoma in Northeast Scotland. Instead the authors were able to conduct a pragmatic study which provided invaluable information to inform the further development of a digital intervention to support TSSE in those diagnosed with cutaneous melanoma. The results are broadly in keeping with what has been published on melanoma recurrence before, 
but the authors were able to identify where such an intervention should be targeted to achieve maximum benefit in a way that previous research has not done.

This sample of patients was small from a relatively small area. The results may not be generalizable. However, two facts mitigate against this limitation. Firstly, in population terms melanoma recurrence is relatively rare and consequently its epidemiology is under-researched. Small studies such as this can signpost the way toward much needed larger studies. Secondly, proportions of follow-up versus self detected melanoma observed here are similar to those previously reported from other regions. This suggests that there is scope for others to add to our findings using similar small samples. A further related limitation is that the sample is dominated by men. Since men are arguably less likely to sustain TSSE this should be borne in mind as a potential confounder for which we have not been adequately able to control. As with all retrospective studies based upon medical records this study was limited by the availability and quality of records. This limitation is, however, common to almost all previous work on the epidemiology of recurrent melanoma, further highlighting the need for larger studies on the epidemiology of melanoma recurrence in future.

\section{Conclusions}

Scheduled follow-up is important and effective in detecting recurrent melanoma particularly in the immediate post diagnostic period. Patients who are within one year of being diagnosed with a primary cutaneous melanoma are significantly less likely to detect their own recurrence, potentially placing them at risk of poorer outcomes. A digital intervention to prompt and support TSSE for people diagnosed with cutaneous melanoma should start immediately a patient enters follow-up. Such an intervention should not be viewed as an alternative to current structured follow-up and may become less necessary over time. An immediate research priority is to develop an effective and user friendly digital application to prompt and support TSSE in those newly diagnosed with cutaneous melanoma. Subsequent research should include a randomised trial to ensure that such an intervention can sustain TSSE in the longer term. Further large-scale research on the epidemiology of recurrent melanoma is also required.

\section{Competing interests}

All authors declare that they have no competing interests to declare.

\section{Authors' contributions}

RA, PW, MN, and PM designed the study. RA and PW collected and managed the data. RA, PW and PM conducted data analysis. RA, PW, SH, MN, PM wrote the manuscript. PM is the guarantor. All authors read and approved the final manuscript.

\section{Acknowledgements}

We wish to acknowledge the funders of the research described here which was supported by the award made by the RCUK Digital Economy programme to the dot.rural Digital Economy Hub; award reference: $\mathrm{EP} / \mathrm{G} 066051 / 1$. We also wish to acknowledge the University of Aberdeen who acted as sponsors for the study.

\section{Author details}

${ }^{1}$ School of Biomedical Sciences, Medical School, Teviot Place, Edinburgh EH8 $9 A G$, UK. ${ }^{2}$ Medical School, University of Aberdeen, Polwarth Building, Foresterhill, Aberdeen AB25 2ZD, UK. ${ }^{3}$ Centre of Academic Primary Care - Division of Applied Health Sciences, University of Aberdeen, Polwarth Building, Foresterhill, Aberdeen AB25 2ZD, UK. ${ }^{4}$ ANCHOR Unit, Aberdeen Royal Infirmary, Foresterhill, Aberdeen AB25 2ZN, UK.

Received: 11 October 2013 Accepted: 27 February 2014

Published: 11 March 2014

\section{References}

1. Erdmann F, Lortet-Tieulent J, Schüz J, Zeeb H, Greinert R, Breitbart BW, Bray F: International trends in the incidence of malignant melanoma 1953-2008 are recent generations at higher or lower risk? Int J Cancer 2013, 132:385-400.

2. Garbe C, Leiter U: Melanoma epidemiology and trends. Clin Dermatol 2009, 27:3-9.

3. Korner $\mathrm{A}$ : Barriers and facilitators of adherence to medical skin self-examination during melanoma follow-up care. BMC Dermatol 2013, 13:3.

4. Scottish Intercollegiate Guidelines Network: Cutaneous Melanoma, Guideline 72. Edinburgh: SIGN; 2003.

5. Ugurel S, Enk A: Skin cancer; follow-up, rehabilitation, palliative and supportive care. J. Dtsch Dermatol. Ges. IOS 2008, 6:492-498.

6. Terushkin V, Halpern AC: Melanoma early detection. Hematol Oncol Clin North Am 2009, 23:481-500.

7. Hall SJ, Samuel LM, Murchie P: Toward shared care for people with cancer: developing the model with patients and GPs. Fam Pract 2011, 28:554-564.

8. Dicker TJ, Kavanagh GM, Herd RM, Ahmad T, McLaren KM, Chetty U, Hunter $\mathrm{JA}$ : A rational approach to melanoma follow-up in patients with primary cutaneous melanoma. Brit J Dermatol 2009, 140:249-254.

9. Meyers MO: Method of detection of initial recurrence of stage II/III cutaneous melanoma: analysis of the utility of follow-up staging. Annal Onco 2009, 16:941-947.

10. Poo-Hwu WJ, Ariyan S, Lamb L, Papac R, Zelterman D, Hu GL, Brown J, Fischer D, Bolgnia J, Buzaid AC: Follow up recommendations for patients with American joint committee of cancer stages I-III malignant melanoma. Cancer 1999, 86:2252-2258.

11. Garbe C: Prospective evaluation of a follow-up schedule in cutaneous melanoma patients: recommendations for an effective follow-up strategy. J Clin Oncol 2003, 21:520-529.

12. Kruijff S, Bastiannet E, Surmeijer AJH, Hoekstra HJ: Detection of melanoma nodal metastases; differences in detection between elderly and young patients do not effect survival. Annal Onco 2010, 17:3008-3014.

13. Moore-Dalal K, Zhou Q, Panageas KS, Brady MS, Jaques DP, Coit DG: Methods of detection of first recurrence in patients with stage $1 / I$ primary cutaneous melanoma after sentinel lymph node biopsy. Annal Onco 2008, 15:2206-2214.

14. Hull P, Piemontesi N, Lichtenwald J: Compliance with self-examination surveillance in patients with melanoma and atypical moles: an anonymous questionnaire study. J Cutan Med Surg 2011, 15:97-102.

15. Korner A, Coroiu A, Martins C, Wang B: Predictors of skin self-examination before and after a melanoma diagnosis; the role of medical advice and patient's level of education. Int Arch Med 2013, 6:8.

16. Janda M, Baade PD, Youl PH, Aitken JF, Whiteman DC, Gordon L, Neale RE: The skin awareness study: promoting thorough skin self-examination for skin cancer among men 50 years or older. Contemp Clin Trials 2009, 31:119-130.

17. Janda M, Neale RE, Youl P, Whiteman DC, Gordon L, Baade PD: Impact of video-based intervention to improve the prevalence of skin self-examinations in men 50 years or older: the randomized skin awareness trial. Arch Dermatol 2011, 147:799-806.

18. Lee $K$, Weinstock M, Risica P: Component of a successful intervention for monthly skin self-examination for early detection of melanoma: the 'check it out' trial. J Am Acad Dermatol 2008, 58:1006-1012. 
19. Oliveria S, Dusza S, Phelan D, Ostroff J, Berwick M, Halpern A: Patient adherence to skin self-examination; effect of nurse intervention with photographs. Am J Prev Med 2004, 26:152-155.

20. Weinstock M, Risica P, Martin R, Rakowski W, Dube C, Berwick M, Goldstein MG, Acharyya S, Lasater T: Melanoma early detection with thorough skin self-examination; The 'Check It Out' Randomized Trial. Am J Prev Med 2007, 32:517-524.

21. Scottish Government: Scottish Index of Multiple Deprivation. 2012. Available: http://www.scotland.gov.uk/Topics/Statistics/SIMD (Accessed 4/9/13).

22. Scottish Government: Scottish Government 2-Fold Urban Rural Classification. 2010. Available: http://www.scotland.gov.uk/Topics/Statistics/About/ Methodology/UrbanRuralClassification (Accessed 4/9/13).

23. Printz C: Target melanoma: skin cancer screenings hold promise to reduce mortality rates but usage of widespread screening lags. Cancer 2008, 119:2359-2360.

24. Leiter U, Marghoob AA, Lasithiotakis K, Eigentler TK, Meier F, Meisner C, Garbe C: Costs of the detection of metastases and follow-up examinations in cutaneous melanoma. Melanoma Res 2009, 19:50-57.

25. Beaver K, Hollingworth W, McDonald R, Dunn G, Tysver-Robinson D, Thomson L, Hindley AC, Susnerwala SS, Luker K: Economic evaluation of a randomized clinical trial of hospital versus telephone follow-up after treatment for breast cancer. Brit J Surg 2009, 96:1406-1415.

26. Stanton AL, Ganz PA, Rowland JH, Meyerowitz BE, Krupnick JL, Sears SR: Promoting adjustment after treatment for cancer. Cancer 2005, 104(11 Suppl):2608-2613.

27. Mackie R, Bray C, Hole D, Morris A, Nicolson M, Evans A, Doherty V, Vestey J: Incidence of and survival from malignant melanoma in Scotland: an epidemiological study. Lancet 2002, 360:587-591.

doi:10.1186/1471-5945-14-4

Cite this article as: Auckland et al:: Exploring patterns of recurrent melanoma in Northeast Scotland to inform the introduction a digital self-examination intervention. BMC Dermatology 2014 14:4.

\section{Submit your next manuscript to BioMed Central and take full advantage of:}

- Convenient online submission

- Thorough peer review

- No space constraints or color figure charges

- Immediate publication on acceptance

- Inclusion in PubMed, CAS, Scopus and Google Scholar

- Research which is freely available for redistribution 